\title{
THE FIXED POINT PROPERTY IN EVERY WEAK HOMOTOPY TYPE
}

\author{
JONATHAN ARIEL BARMAK ${ }^{\dagger}$
}

\begin{abstract}
We prove that for any connected compact CW-complex $K$ there exists a space $X$ weak homotopy equivalent to $K$ which has the fixed point property, that is, every continuous map $X \rightarrow X$ has a fixed point. The result is known to be false if we require $X$ to be a polyhedron. The space $X$ we construct is a non-Hausdorff space with finitely many points.
\end{abstract}

A topological space $X$ has the fixed point property if every continuous map $f: X \rightarrow X$ has a fixed point. We will prove the following

Theorem 1. Let $K$ be a connected compact $C W$-complex. Then there exists a topological space $X$ weak homotopy equivalent to $K$ with the fixed point property.

If we require $X$ to be a polyhedron, the result is known to be false. Though the fixed point property is not a homotopy invariant, every polyhedron homotopy equivalent to a sphere lacks the fixed point property (see [6, Theorem 7.1], [8] or the proof of [11, Theorem]). The space $X$ we find has finitely many points. Therefore, we are also proving the following result. The homotopy type of any connected compact $\mathrm{CW}$-complex can be realized by the order complex of a finite partially ordered set with the fixed point property.

A simplicial complex $K$ has the fixed simplex property if for every simplicial map $f$ : $K \rightarrow K$ there exists a simplex $\sigma \in K$ such that $f(\sigma)=\sigma$ or, equivalently, if every simplicial endomorphism of $K$ fixes a point of the realization of $K$. The spheres $S^{n}$ do not have the fixed point property, but they do have triangulations with the fixed simplex property provided that $n \geq 2$ (see Proposition 3 ). We will show that for every simply connected compact polyhedron $K$ there exists a finite simplicial complex $L$ homotopy equivalent to $K$ with the fixed simplex property. Then the finite topological space $\mathcal{X}(L)$ associated to $L$ has the fixed point property. This will prove Theorem 1 for simply connected complexes. If $K$ is not simply connected we will be able to modify the construction above to obtain a finite model of $K$ with the fixed point property but it will not be the poset of faces of a complex.

We sketch in a few lines the idea of the construction of $L$ from $K$ and the main parts of the proof. We first consider integer homology classes $\alpha_{k, l} \in H_{k}(K)$ which are a basis of the rational $k$-homology of $K$ and then realize each $\alpha_{k, l}$ as the image of the fundamental class $\left[M_{k, l}\right]$ of a $k$-dimensional oriented pseudomanifold through a map $M_{k, l} \rightarrow K$. We construct $L$ as follows. We find a sufficiently fine ( $j$-th barycentric) subdivision $K^{j}$ of $K$ and attach to $K^{j}$ mapping cylinders of the maps $M_{k, l}^{j} \rightarrow K^{j}$ and of approximations to the

2010 Mathematics Subject Classification. 55M20, 55U10, 54H25, 06A07.

Key words and phrases. Fixed point property, simplicial complexes, weak homotopy types, Lefschetz fixed point theorem, finite topological spaces.

$\dagger$ Researcher of CONICET. Partially supported by grants UBACyT 20020100300043, CONICET PIP 112-201101-00746 and ANPCyT PICT-2011-0812. 
identities $M_{k, l}^{j} \rightarrow M_{k, l}^{r}(r<j)$. In this way we manage to concentrate the homology classes $\alpha_{k, l}$ in "few" simplices, those of $M_{k, l}^{r}$. The complex $L$ satisfies this singular property: if $c=\sum t_{i} \sigma_{i} \in C_{k}(L)$ is a cycle such that $\sum\left|t_{i}\right|$ is less than or equal to the number of $k$ simplices in $M_{k, l}^{r}$ and the $\left(\alpha_{k, l} \otimes 1_{\mathbb{Q}}\right)$-coordinate of $[c] \in H_{k}(L ; \mathbb{Q})$ is different from zero, then $c$ is, up to sign, the fundamental cycle of $M_{k, l}^{r}$. Therefore if $f$ is a simplicial endomorphism of $L$, one of the following holds: (1) the matrices of $f_{*}: H_{k}(L ; \mathbb{Q}) \rightarrow H_{k}(L ; \mathbb{Q})$ in the basis $\left\{\alpha_{k, l} \otimes 1_{\mathbb{Q}}\right\}_{l}$ have all the diagonal zero for each $k \geq 2$, in which case the Lefschetz fixed point theorem gives us a fixed simplex, or (2) the map $f$ maps one of the $M_{k, l}^{r}$ into itself and $\left.f\right|_{M_{k, l}^{r}}: M_{k, l}^{r} \rightarrow M_{k, l}^{r}$ is a simplicial automorphism. The argument is then complete if we show that the pseudomanifolds $M_{k, l}^{r}$ can be assumed to be asymmetric, in the sense that every automorphism fixes a vertex.

The following notions are a rigid version of Gromov's simplicial volume and the $\ell^{1}$ norm. Let $C$ be a finitely generated free $\mathbb{Z}$-module with a fixed basis $\left\{b_{1}, b_{2}, \ldots, b_{r}\right\}$. The norm $\|c\|$ of an element $c=\sum t_{i} b_{i} \in C$ is $\sum\left|t_{i}\right|$. If $f: C \rightarrow C^{\prime}$ is a morphism between finitely generated free $\mathbb{Z}$-modules (each of them with a chosen basis), the norm of $f$ is $\|f\|=\max _{c \neq 0} \frac{\|f(c)\|}{\|c\|}$. Note that $\|f\|$ is well-defined since $\|f(c)\| \leq\|c\| \max _{i}\left\|f\left(b_{i}\right)\right\|$, where $\left\{b_{i}\right\}_{i}$ is the chosen basis of $C$. If $C=0$ define $\|f\|=0$. For a composition $f g$ we have $\|f g\| \leq\|f\|\|g\|$.

Let $C_{*}$ be a finitely generated free chain complex with a given basis for each $C_{k}$. The norm of $\alpha \in H_{k}(C)$ is $\|\alpha\|=\min \{\|c\| \mid[c]=\alpha\}$. Here $[c]$ denotes the class of a cycle $c$ in homology.

When $K$ is a finite simplicial complex we will always consider the chain complex $C_{*}(K)$ with the usual basis for $C_{k}(K)$ given by one oriented $k$-simplex $\left[v_{0}, v_{1}, \ldots, v_{k}\right]$ for each $k$-simplex $\left\{v_{0}, v_{1}, \ldots, v_{k}\right\} \in K$. We denote by $H_{*}(K)$ the simplicial homology of $K$ with integer coefficients.

If $M$ is a closed $n$-dimensional oriented pseudomanifold, the norm $\|[M]\| \in H_{n}(M)$ of its fundamental class is the number of $n$-simplices in $M$.

If $\varphi: K \rightarrow L$ is a simplicial map between finite simplicial complexes, $\varphi_{*}: C_{*}(K) \rightarrow$ $C_{*}(L)$ maps an oriented $k$-simplex $\left[v_{0}, v_{1}, \ldots, v_{k}\right]$ to $\left[\varphi\left(v_{0}\right), \varphi\left(v_{1}\right), \ldots, \varphi\left(v_{k}\right)\right]$ if $\varphi\left(v_{i}\right) \neq$ $\varphi\left(v_{j}\right)$ for $i \neq j$ and to 0 otherwise. Therefore $\varphi_{k}: C_{k}(K) \rightarrow C_{k}(L)$ has norm at most 1.

If $L$ is a finite simplicial complex and $K$ is a subdivision of $L$, the subdivision operator $\lambda: C_{*}(L) \rightarrow C_{*}(K)$ is a homotopy inverse to the chain map induced by any simplicial approximation to the identity and maps a $k$-simplex $\sigma \in L$ into a signed sum of all the $k$-simplices of $K$ contained in $\sigma$. Therefore, the norm of $\lambda_{k}: C_{k}(L) \rightarrow C_{k}(K)$ is the maximum number of $k$-simplices in which a $k$-simplex of $L$ is subdivided. In particular, when $K$ is the first barycentric subdivision $L^{\prime}$ of $L,\left\|\lambda_{k}\right\|=(k+1)$ ! if $\operatorname{dim} L \geq k$. In this case, if $\alpha \in H_{k}(L),\left\|\lambda_{*}(\alpha)\right\| \leq\left\|\lambda_{k}\right\|\|\alpha\| \leq(k+1) !\|\alpha\|$. In general the equality $\left\|\lambda_{*}(\alpha)\right\|=(k+1) !\|\alpha\|$ does not hold if $k<\operatorname{dim}(L)$.

Let $K$ be a finite simplicial complex. The barycenter of a simplex $\sigma \in K$ will be denoted by $b(\sigma)$ or $\hat{\sigma}$. The simplices of $K^{\prime}$ are then the sets $\left\{\hat{\sigma}_{0}, \hat{\sigma}_{1}, \ldots, \hat{\sigma}_{k}\right\}$ such that $\sigma_{i} \subsetneq \sigma_{i+1}$ for every $i$. Given a simplicial map $\varphi: K \rightarrow L$, we denote by $\varphi^{\prime}: K^{\prime} \rightarrow L^{\prime}$ the map $b(\sigma) \mapsto b(\varphi(\sigma))$ and in general $\varphi^{j}: K^{j} \rightarrow L^{j}$ is the map induced in the $j$-th barycentric subdivisions.

In [1, Example 2.4], Baclawski and Björner construct a finite model of $S^{2}$ with the fixed point property. The following definition is inspired by their example. 
Definition 2. We will say that a complex $K$ is asymmetric if there exists a vertex $v \in K$ which is fixed by every simplicial automorphism of $K$.

Proposition 3. Let $M$ be an $n$-dimensional pseudomanifold with $n \geq 2$. Then there exists a subdivision $L$ of $M$ such that the $j$-th barycentric subdivision $L^{j}$ of $L$ is asymmetric for every $j \geq 0$.

Proof. Given a complex $K$ and a simplex $\sigma \in K$, we denote by $\operatorname{deg}_{K}(\sigma)$ the number of maximal simplices of $K$ containing $\sigma$. Then $\operatorname{deg}_{K}(\sigma)$ is the number of maximal simplices in the link $\operatorname{lk}_{K}(\sigma)$ if $\sigma$ is not maximal, and 1 if $\sigma$ is maximal in $K$. Define $d(K)=$ $\max _{\sigma \in K} \operatorname{deg}_{K}(\sigma)=\max _{v \in K} \operatorname{deg}_{K}(v)$ where the second maximum is taken over all the vertices $v$ of $K$. It is not hard to see that there exists a subdivision $L$ of $M$ which contains a vertex $v_{0}$ such that $\operatorname{deg}_{L}(v)<\operatorname{deg}_{L}\left(v_{0}\right)$ for any other $v \in L$. Then clearly $L$ is asymmetric since the degree deg is preserved by automorphisms of $L$ and thus $v_{0}$ is fixed by any such an automorphism. Moreover, we will show that $\operatorname{deg}_{L^{\prime}}(v)<\operatorname{deg}_{L^{\prime}}\left(v_{0}\right)$ for every vertex $v_{0} \neq v \in L^{\prime}$. It follows by induction that $L^{j}$ is asymmetric for each $j \geq 0$.

Let $v \in L^{\prime}, v=b(\sigma)$ where $\sigma$ is a simplex of $L$. Let $k=\operatorname{dim} \sigma$. A maximal simplex of $\mathrm{lk}_{L^{\prime}}(b(\sigma))$ is obtained by choosing a chain $\sigma^{0}<\sigma^{1}<\ldots<\sigma^{k-1}$ of proper faces of $\sigma$ and a chain $\sigma^{k+1}<\sigma^{k+2}<\ldots<\sigma^{n}$ of simplices containing $\sigma$. There are $(k+1)$ possible choices for $\sigma^{k-1}, k$ for $\sigma^{k-2}, \ldots, 2$ for $\sigma^{0}$. On the other hand there are $\operatorname{deg}_{L}(\sigma)$ choices for $\sigma^{n},(n-k)$ for $\sigma^{n-1},(n-k-1)$ for $\sigma^{n-2}, \ldots, 2$ for $\sigma^{k+1}$. Therefore

$$
\operatorname{deg}_{L^{\prime}}(b(\sigma))=(k+1) !(n-k) ! \operatorname{deg}_{L}(\sigma) .
$$

-If $k=n, \operatorname{deg}_{L^{\prime}}(b(\sigma))=(n+1) !<n ! d(L)$ since $d(L)=n+1$ only when the pseudomanifold is isomorphic to the boundary of an $(n+1)$-simplex, which is not the case since $L$ is asymmetric.

-If $k=n-1, \operatorname{deg}_{L^{\prime}}(b(\sigma))=n ! \operatorname{deg}_{L}(\sigma)=2 n !<n ! d(L)$.

-If $1 \leq k \leq n-2,\left(\begin{array}{l}n \\ k\end{array}\right) \geq n$, so $\operatorname{deg}_{L^{\prime}}(b(\sigma)) \leq(n-1) !(k+1) \operatorname{deg}_{L}(\sigma)<n ! d(L)$.

-If $k=0, \sigma=v$ is a vertex of $L$ and $\operatorname{deg}_{L^{\prime}}(v)=n ! \operatorname{deg}_{L}(v)$, which is strictly smaller than $n ! d(L)$ if $v \neq v_{0}$ and which is $n ! \operatorname{deg}_{L}\left(v_{0}\right)=n ! d(L)$ if $v=v_{0}$. Then $d\left(L^{\prime}\right)=n ! d(L)$ and this degree is only achieved by $v_{0}$.

Lemma 4. Let $\tau$ be a simplex. Let $\sigma_{0}, \sigma_{1}, \ldots, \sigma_{k}$ be faces of $\tau$ such that for every $0 \leq$ $i, j \leq k$ one of the following holds:

(1) $\sigma_{i} \subseteq \sigma_{j}$.

(2) $\sigma_{j} \subseteq \sigma_{i}$.

(3) $\sigma_{i} \cap \sigma_{j}=\emptyset$.

Then the convex hull of $\left\{\hat{\sigma}_{0}, \hat{\sigma}_{1}, \ldots, \hat{\sigma}_{k}\right\}$ is a subcomplex of $\tau^{\prime}$.

Proof. We proceed by induction in the number of pairs $i, j$ satisfying (3). If (1) or (2) holds for every $i, j$ then the convex hull $S$ of $\left\{\hat{\sigma}_{0}, \hat{\sigma}_{1}, \ldots, \hat{\sigma}_{k}\right\}$ is a simplex of $\tau^{\prime}$. Otherwise take $i, j$ such that $\sigma=\sigma_{i} \cup \sigma_{j}$ has maximum cardinality among all the pairs satisfying (3). Since $\hat{\sigma}$ is a convex combination of $\hat{\sigma}_{i}$ and $\hat{\sigma}_{j}$, by induction it suffices to prove that $\left\{\sigma, \sigma_{0}, \sigma_{1}, \ldots, \sigma_{i-1}, \sigma_{i+1}, \ldots, \sigma_{k}\right\}$ and $\left\{\sigma, \sigma_{0}, \sigma_{1}, \ldots, \sigma_{j-1}, \sigma_{j+1}, \ldots, \sigma_{k}\right\}$ satisfy the hypothesis of the lemma. Let $0 \leq l \leq k, i \neq l \neq j$. We have to verify that $\sigma$ and $\sigma_{l}$ are comparable or disjoint. If $(i, l)$ and $(j, l)$ satisfy (3), then $\sigma$ and $\sigma_{l}$ are disjoint. Suppose $(i, l)$ satisfies (3) and $\sigma_{j}$ is comparable with $\sigma_{l}$. By the choice of $i$ and $j, \sigma_{j}$ cannot be a proper face of $\sigma_{l}$. Then $\sigma_{l} \subseteq \sigma_{j} \subseteq \sigma$ so $\sigma_{l}$ and $\sigma$ are comparable. By symmetry it only remains to 
analyze the case that $\sigma_{l}$ is comparable with both $\sigma_{i}$ and $\sigma_{j}$. If $\sigma_{l}$ is a face of any of them, then it is a face of $\sigma$. If $\sigma_{i}$ and $\sigma_{j}$ are faces of $\sigma_{l}$, then so is $\sigma$.

Remark 5. In the conditions of Lemma 4 , note that if the convex hull $S$ of $\left\{\hat{\sigma}_{0}, \hat{\sigma}_{1}, \ldots, \hat{\sigma}_{k}\right\}$ is a $k$-dimensional subcomplex of $\tau^{\prime}$, then it contains at most $(k+1)$ ! many $k$-simplices of $\tau^{\prime}$. Moreover, if the equality holds then all the pairs $i, j$ satisfy condition (3). The proof of Lemma 4 shows that the vertices of $S \leq \tau^{\prime}$ are barycenters of unions of simplices $\sigma_{i}$. Therefore, the $k$-simplices of $\tau^{\prime}$ contained in $S$ are of the form $\left\{\hat{\tau}_{0}, \hat{\tau}_{1}, \ldots, \hat{\tau}_{k}\right\}$ where $\tau_{l}=\bigcup_{i \in A_{l}} \sigma_{i}, A_{l} \subseteq[0, k]$, and $\tau_{l} \subsetneq \tau_{l+1}$. Moreover, we can assume that $A_{l} \subsetneq A_{l+1}$. Since there are only $(k+1)$ ! sequences $\emptyset \neq A_{0} \subsetneq A_{1} \subsetneq \ldots \subsetneq A_{k} \subseteq[0, k], S$ is decomposed in at most $(k+1)$ ! many $k$-simplices. If $\sigma_{i} \subseteq \sigma_{j}$ for some $i \neq j$ then half of these sequences do not give a $k$-simplex, so $S$ is decomposed in at most $\frac{(k+1) !}{2}$ many $k$-simplices.

Let $\varphi: K \rightarrow L$ be a simplicial map between finite simplicial complexes. We will work with the following version of the simplicial mapping cylinder $Z_{\varphi}$ of $\varphi$. First we choose a total ordering in the set of vertices of $K$. The vertex set of $Z_{\varphi}$ is the disjoint union of the vertex set of $K$ and of $L$. The simplices of the cylinder are the simplices of $L$ together with sets of the form $\left\{v_{0}, v_{1}, \ldots, v_{l}, \varphi\left(v_{l+1}\right), \varphi\left(v_{l+2}\right), \ldots, \varphi\left(v_{m}\right)\right\}$ where $\left\{v_{0}, v_{1}, \ldots, v_{m}\right\}$ is a simplex of $K$ and $v_{i} \leq v_{i+1}$ for every $i$ ( $v_{l}$ could be equal to $v_{l+1}$ ).

There is a simplicial retraction $p: Z_{\varphi} \rightarrow L$ of the canonical inclusion $j: L \rightarrow Z_{\varphi}$ defined by $p(v)=\varphi(v)$ if $v \in K$. Therefore $p i=\varphi$ where $i$ denotes the canonical inclusion of $K$ into the cylinder. The composition $j p$ lies in the same contiguity class as the identity $1_{Z_{\varphi}}$, so $p_{*}: C_{*}\left(Z_{\varphi}\right) \rightarrow C_{*}(L)$ is a homotopy equivalence [10, p.151].

Suppose $K$ is a subdivision of a complex $L$ and that $\psi: K \rightarrow L$ is a simplicial approximation to the identity. In other words, $\psi$ is a vertex map which maps each vertex $v \in K$ to any vertex $w \in L$ of the unique open simplex of $L$ containing $v$. In this case $\psi_{*}: C_{*}(K) \rightarrow C_{*}(L)$ is a homotopy equivalence. Since $p_{*}: C_{*}\left(Z_{\psi}\right) \rightarrow C_{*}(L)$ is a homotopy equivalence and $\psi=p i, i_{*}: C_{*}(K) \rightarrow C_{*}\left(Z_{\psi}\right)$ is a homotopy equivalence. Since $C_{*}(K)$ is a subcomplex of $C_{*}\left(Z_{\psi}\right)$, it is known that there exists a retraction $r: C_{*}\left(Z_{\psi}\right) \rightarrow C_{*}(K)$. However, we need to control the norm $\left\|r_{k}\right\|$ of each $r_{k}: C_{k}\left(Z_{\psi}\right) \rightarrow C_{k}(K)$. We will prove that for barycentric subdivisions $K=L^{\prime}$ there is a retraction $r$ such that $\left\|r_{k}\right\| \leq(k+1)$ !. It is not true that this inequality holds for any retraction $r$.

Lemma 6. Let $K$ be a finite simplicial complex. Then there exists an ordering of the vertices of $K^{\prime}$, a simplicial approximation to the identity $\psi: K^{\prime} \rightarrow K$ and a retraction $r: C_{*}\left(Z_{\psi}\right) \rightarrow C_{*}\left(K^{\prime}\right)$ satisfying the following

(1) If $S$ is a $k$-simplex of $Z_{\psi}$, then $\left\|r_{k}(S)\right\| \leq(k+1)$ !.

(2) If $S$ is a k-simplex of $Z_{\psi}$ with $k \geq 1$ such that $\left\|r_{k}(S)\right\|=(k+1)$ !, then $S \in K$.

Proof. Order the vertices $\hat{\sigma}$ of $K^{\prime}$ in such a way that $\hat{\sigma}<\hat{\tau} \operatorname{implies} \operatorname{dim}(\sigma) \geq \operatorname{dim}(\tau)$. Let $\psi: K^{\prime} \rightarrow K$ be any approximation to the identity. In other words, if $\hat{\sigma}$ is a vertex of $K^{\prime}$, then $\psi(\hat{\sigma}) \in \sigma$. A $k$-simplex of $Z_{\psi}$ is of the form $S=\left\{\hat{\sigma}_{0}, \hat{\sigma}_{1}, \ldots, \hat{\sigma}_{l}, \psi\left(\hat{\sigma}_{l+1}\right), \psi\left(\hat{\sigma}_{l+2}\right), \ldots\right.$, $\left.\psi\left(\hat{\sigma}_{m}\right)\right\}$ where $l \geq-1, m \geq l$ and $\sigma_{i+1} \subseteq \sigma_{i}$ for all $0 \leq i \leq m-1$ (the simplices of $K$ are included in these). We can consider $S$ as a set of vertices of $K^{\prime}$ identifying $v_{i}=\psi\left(\hat{\sigma}_{i}\right)$ with the barycenter of $\left\{v_{i}\right\}$. The hypothesis of Lemma 4 is satisfied. For any $0 \leq i, j \leq l, \sigma_{i}$ and $\sigma_{j}$ are comparable; if $l+1 \leq i, j \leq m,\left\{v_{i}\right\}$ and $\left\{v_{j}\right\}$ are disjoint or equal; if $0 \leq i \leq l$ and $l+1 \leq j \leq m$, then $\sigma_{i} \supseteq \sigma_{j} \supseteq\left\{v_{j}\right\}$. Thus, by Lemma 4, the convex hull of $S$ is a subcomplex $\Phi(S)$ of $K^{\prime}$. The application $\Phi$ defines an acyclic carrier from $Z_{\psi}$ to $K^{\prime}$. Let 
$r: C_{*}\left(Z_{\psi}\right) \rightarrow C_{*}\left(K^{\prime}\right)$ be a chain map carried by $\Phi$. Note that $r$ is uniquely determined by $\Phi$ since for a $k$-simplex $S \in Z_{\psi}, \Phi(S)$ is $j$-dimensional with $j \leq k$. Thus, any homotopy $F: C_{*}\left(Z_{\psi}\right) \rightarrow C_{*+1}\left(K^{\prime}\right)$ carried by $\Phi$, given by the Acyclic carrier theorem, must be trivial.

If $S \in Z_{\psi}$ is a $k$-simplex such that $\operatorname{dim} \Phi(S)<k$, then $r(S) \in C_{k}(\Phi(S))=0$ is trivial. Otherwise $\operatorname{dim} \Phi(S)=k$ and then $\Phi(S)$ is a subdivision of $S$ (considered as a set of $k+1$ affinely independent vertices of $\left.K^{\prime}\right)$. One has then the subdivision operator $\lambda: C_{*}(S) \rightarrow$ $C_{*}(\Phi(S))$. Since for each $j$-face $\widetilde{S}$ of $S, \Phi(\widetilde{S})$ is a $j$-dimensional subcomplex, the acyclic carrier $\Phi$ when restricted to $C_{*}(S)$ is the usual subdivision carrier $\Phi(\widetilde{S})=K^{\prime}(\widetilde{S})$. Thus $\lambda,\left.r\right|_{C_{*}(S)}: C_{*}(S) \rightarrow C_{*}(\Phi(S))$ are carried by the same acyclic carrier $\Phi$ and by the same argument as before, they coincide. Hence $\left\|r_{k}(S)\right\|=\left\|\lambda_{k}(S)\right\|$ is the number of $k$-simplices in $\Phi(S)$ which is at most $(k+1)$ ! by Remark 5 .

Cearly $r: C_{*}\left(Z_{\psi}\right) \rightarrow C_{*}(K)$ is a retraction since for $S \in K^{\prime}$ we have $\Phi(S)=S$ and $\lambda(S)=S$.

Finally, suppose $S=\left\{\hat{\sigma}_{0}, \hat{\sigma}_{1}, \ldots, \hat{\sigma}_{l}, \psi\left(\hat{\sigma}_{l+1}\right), \psi\left(\hat{\sigma}_{l+2}\right), \ldots, \psi\left(\hat{\sigma}_{m}\right)\right\}$ is a $k$-simplex of $Z_{\psi}$ with $k \geq 1$. If $l \geq 0, \sigma_{0}$ is comparable with each $\sigma_{j}$ and each $\left\{v_{j}\right\}$. By Remark $5, S$ is subdivided in less than $(k+1) ! k$-simplices of $K^{\prime}$ so $\left\|r_{k}(S)\right\|<(k+1)$ !. This proves the second assertion of the lemma.

Remark 7. It is well-known that every singular $k$-homology class $\alpha$ of a space $X$ can be realized by a disjoint union $\sqcup M_{i}$ of closed $k$-dimensional oriented pseudomanifolds, meaning that there is a continuous map $f: \sqcup M_{i} \rightarrow X$ such that $\sum f_{*}\left(\left[M_{i}\right]\right)=\alpha$ (see $[5$, p.108] for example). Then for every simplicial homology class $\alpha \in H_{k}(K)$ of a simplicial complex $K$ there exists a simplicial map $\varphi: \sqcup M_{i} \rightarrow K$ from a disjoint union of closed oriented pseudomanifolds such that $\sum \varphi_{*}\left(\left[M_{i}\right]\right)=\alpha$.

Theorem 8. Let $K$ be a finite simplicial complex which is simply connected or, more generally, such that $H_{1}(K)=0$. Then there exists a finite simplicial complex L homotopy equivalent to $K$ with the fixed simplex property.

\section{Proof. First part: Construction of $L$}

Let $n=\operatorname{dim}(K)$. For each $2 \leq k \leq n$ let $d_{k}$ be the rank of $H_{k}(K ; \mathbb{Q})$. Take for each $2 \leq k \leq n$ homology classes $\alpha_{k, 1}, \alpha_{k, 2}, \ldots, \alpha_{k, d_{k}} \in H_{k}(K)$ such that $\left\{\alpha_{k, 1} \otimes 1_{\mathbb{Q}}, \alpha_{k, 2} \otimes\right.$ $\left.1_{\mathbb{Q}}, \ldots, \alpha_{k, d_{k}} \otimes 1_{\mathbb{Q}}\right\}$ is a basis of $H_{k}(K ; \mathbb{Q})$. By Remark 7 each $\alpha_{k, l}$ can be realized by a disjoint union of closed oriented pseudomanifolds. Moreover, by changing the $\alpha_{k, l}$ 's if needed we can assume that each of them is realized by a single pseudomanifold. For each $k \geq 2$ and $1 \leq l \leq d_{k}$ let $M_{k, l}$ be a $k$-dimensional oriented pseudomanifold and let $\varphi_{k, l}: M_{k, l} \rightarrow K$ be a simplicial map such that $\left(\varphi_{k, l}\right)_{*}\left(\left[M_{k, l}\right]\right)=\alpha_{k, l}$. By Proposition 3 we can assume that $M_{k, l}$ is asymmetric for each $k, l$ as well as all their iterated barycentric subdivisions.

We define an increasing sequence $s_{1}, s_{2}, s_{3}, \ldots, s_{n}$ of non-negative integers as follows. Let $s_{1}=0$. Let $N_{2, l}=\left\|\left[M_{2, l}\right]\right\|$ be the number of 2 -simplices in $M_{2, l}$ for each $1 \leq l \leq d_{2}$ and let $N_{2}=\max _{l} N_{2, l}$. If $P$ is any finite simplicial complex, the cover $\mathcal{U}$ of $P$ given by the open $\operatorname{stars~}_{\operatorname{st}_{P}}(v)$ of the vertices of $P$ has a Lebesgue number $\delta>0$. Therefore, there exists a positive integer $s_{2}$ such that for each $s \geq s_{2}$, every connected subcomplex of $P^{s}$ generated by at most $N_{2}$ many simplices is contained in an element of $\mathcal{U}$, and in particular in a contractible subcomplex of $P$. We take $s_{2}$ in such a way that the assertion above holds for $P$ when $P$ is any $M_{k, l}$ with $k \geq 3$ and $1 \leq l \leq d_{k}$. 
Now let $N_{3, l}=\left\|\left[M_{3, l}^{s_{2}}\right]\right\|$ for each $1 \leq l \leq d_{3}$ and let $N_{3}=\max N_{3, l}$. Take $s_{3} \geq s_{2}$ such that for each $s \geq s_{3}, k \geq 4$ and $1 \leq l \leq d_{k}$, every connected subcomplex of $M_{k, l}^{s}$ generated by at most $N_{3}$ many simplices is contained in a contractible subcomplex.

In general, suppose $s_{2}, s_{3}, \ldots, s_{m}$ are defined, with $m \leq n-2$. Then define $N_{m+1, l}=$ $\left\|\left[M_{m+1, l}^{s_{m}}\right]\right\|$ for each $1 \leq l \leq d_{m+1}$ and let $N_{m+1}=\max _{l} N_{m+1, l}$. Take $s_{m+1} \geq s_{m}$ such that for each $s \geq s_{m+1}$, every connected subcomplex of $M_{k, l}^{s}$ generated by at most $N_{m+1}$ many simplices is contained in a contractible subcomplex for each $k \geq m+2$ and $1 \leq l \leq d_{k}$.

Finally define $N_{n, l}=\left\|\left[M_{n, l}^{s_{n-1}}\right]\right\|, N_{n}=\max _{l} N_{n, l}, N=\max _{2 \leq k \leq n} N_{k}$ and take $s_{n} \geq s_{n-1}$ such that for each $s \geq s_{n}$, any connected subcomplex of $\bar{K}^{s}$ generated by at most $N$ simplices is contained in a contractible subcomplex.

We now define for each $k \geq 2$ and $1 \leq l \leq d_{k}$ a cylinder $C_{k, l}$ which will be attached to $K^{s_{n}}$. Each $C_{k, l}$ consists of three parts. The first one is $C_{k, l}^{a}=Z_{\varphi_{k, l}^{s_{n}}}$, the cylinder of $\varphi_{k, l}^{s_{n}}: M_{k, l}^{s_{n}} \rightarrow K^{s_{n}}$ (see Figure 1). The second part $C_{k, l}^{b}$ is constructed as follows. We glue $N$ cylinders $Z_{1_{M_{k, l}^{s_{n}}}}$ of the identity $1_{M_{k, l}^{s_{n}}}: M_{k, l}^{s_{n}} \rightarrow M_{k, l}^{s_{n}}$, the second base of one with the first base of the following, to build a long cylinder $C_{k, l}^{b}$ with both bases equal to $M_{k, l}^{s_{n}}$. The last part $C_{k, l}^{c}$ is the union of $s_{n}-s_{k-1}$ mapping cylinders. For each $s_{k-1}<m \leq s_{n}$ there is a simplicial approximation to the identity $\psi_{k, l, m}: M_{k, l}^{m} \rightarrow M_{k, l}^{m-1}$ and a retraction $R_{m}=R_{k, l, m}: C_{*}\left(Z_{\psi_{k, l, m}}\right) \rightarrow C_{*}\left(M_{k, l}^{m}\right)$ satisfying properties (1) and (2) in the statement of Lemma 6 . When we glue the cylinders $Z_{\psi_{k, l, m}}$, identifying a base of one with a base of the following, we obtain a cylinder $C_{k, l}^{c}$ with one base equal to $M_{k, l}^{s_{n}}$ and the other equal to $M_{k, l}^{s_{k-1}}$. Finally we glue $C_{k, l}^{a}$ with one extreme of $C_{k, l}^{b}$ and $C_{k, l}^{c}$ with the other extreme of $C_{k, l}^{b}$. This is $C_{k, l}$.

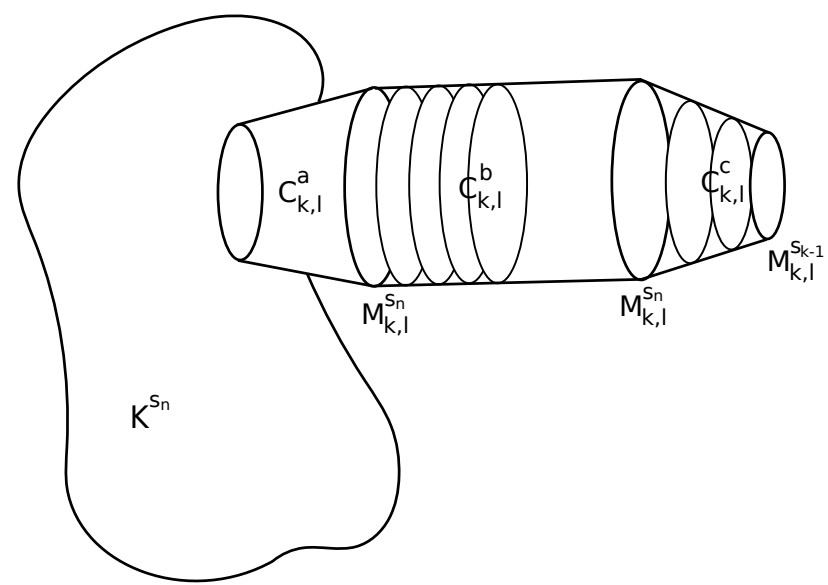

Figure 1. The cylinder $C_{k, l}$, union of $C_{k, l}^{a}, C_{k, l}^{b}$ and $C_{k, l}^{c}$.

Let $L$ be the union of all the cylinders $C_{k, l}$ for $k \geq 2$ and $1 \leq l \leq d_{k}$, all intersecting in $K^{s_{n}}$. Each $C_{k, l}^{c}$ deformation retracts to $M_{k, l}^{s,}, C_{k, l}^{b}$ deformation retracts to $M_{k, l}^{s_{n}}$ and $C_{k, l}^{a}$ deformation retracts to $K^{s_{n}}$. Therefore $L$ is homotopy equivalent to $K$. We will show that $L$ has the fixed simplex property. 


\section{Second part: $L$ has the fixed simplex property}

Let $i_{k, l}: M_{k, l}^{s_{k-1}} \hookrightarrow L$ be the inclusion map in the free extreme of $C_{k, l}^{c}$. Note that $\left(i_{k, l}\right)_{*}\left[M_{k, l}^{s_{k-1}}\right]=i_{*}\left(\varphi_{k, l}^{s_{n}}\right)_{*}\left[M_{k, l}^{s_{n}}\right]=i_{*} \lambda_{*}\left(\alpha_{k, l}\right)$, where $i: K^{s_{n}} \hookrightarrow L$ is the inclusion. Hence, $\mathcal{B}_{k}=\left\{\left(i_{k, l}\right)_{*}\left(\left[M_{k, l}^{s_{k-1}}\right]\right) \otimes 1_{\mathbb{Q}}\right\}_{l}$ is a basis of $H_{k}(L ; \mathbb{Q})$.

Let $f: L \rightarrow L$ be a simplicial map. We study for each $k$ the matrix of $f_{*}: H_{k}(L ; \mathbb{Q}) \rightarrow$ $H_{k}(L ; \mathbb{Q})$ in the basis $\mathcal{B}_{k}$. Since $M_{k, l}^{s_{k-1}}$ has $N_{k, l} \leq N_{k}$ many $k$-simplices, $f\left(M_{k, l}^{s_{k-1}}\right)$ lies in a connected subcomplex of $L$ generated by at most $N_{k}$ simplices. Since each $C_{k^{\prime}, l^{\prime}}^{b}$ is constructed gluing $N \geq N_{k}$ cylinders then one of the following holds:

(1) $f\left(M_{k, l}^{s_{k-1}}\right) \subseteq \bigcup_{k^{\prime}, l^{\prime}}\left(C_{k^{\prime}, l^{\prime}}^{a} \cup C_{k^{\prime}, l^{\prime}}^{b}\right)$ or

(2) $f\left(M_{k, l}^{s_{k-1}}\right) \subseteq C_{k^{\prime}, l^{\prime}}^{b} \cup C_{k^{\prime}, l^{\prime}}^{c}$ for some $k^{\prime}, l^{\prime}$.

In the first case, call $C^{a b}=\bigcup_{k^{\prime}, l^{\prime}}\left(C_{k^{\prime}, l^{\prime}}^{a} \cup C_{k^{\prime}, l^{\prime}}^{b}\right)$. Just as $L$, the complex $C^{a b}$ deformation

retracts to $K^{s_{n}}$, but in contrast to $L$, for $C^{a b}$ the retraction $r^{a b}: C^{a b} \rightarrow K^{s_{n}}$ may be taken simplicial. Since we are assuming $f i_{k, l}: M_{k, l}^{s_{k-1}} \rightarrow C^{a b}, r^{a b} f i_{k, l}\left(M_{k, l}^{s_{k-1}}\right)$ is contained in a connected subcomplex of $K^{s_{n}}$ generated by $N_{k, l} \leq N_{k}$ simplices. By the choice of $s_{n}$, this connected subcomplex lies in a contractible subcomplex of $K^{s_{n}}$, and then $r_{*}^{a b}\left(f i_{k, l}\right)_{*}\left[M_{k, l}^{s_{k-1}}\right]=0 \in H_{k}\left(K^{s_{n}}\right)$. Since $r^{a b}$ is a homotopy equivalence $\left(f i_{k, l}\right)_{*}\left[M_{k, l}^{s_{k-1}}\right]=$ $0 \in H_{k}\left(C^{a b}\right)$, and then $f_{*}\left(i_{k, l}\right)_{*}\left[M_{k, l}^{s_{k-1}}\right]=0 \in H_{k}(L)$. In this case the $l$-th column of the matrix of $f_{*}$ is zero.

Assume then that (2) holds. Call $C_{k^{\prime}, l^{\prime}}^{b c}=C_{k^{\prime}, l^{\prime}}^{b} \cup C_{k^{\prime}, l^{\prime}}^{c}$ and suppose that $f i_{k, l}\left(M_{k, l}^{s_{k-1}}\right) \subseteq$ $C_{k^{\prime}, l^{\prime}}^{b c}$. The complex $C_{k^{\prime}, l^{\prime}}^{b c}$ deformation retracts to $M_{k^{\prime}, l^{\prime}}^{s_{k^{\prime}}-1}$ by a simplicial retraction $r=$ $r_{k^{\prime}, l^{\prime}}^{b c}: C_{k^{\prime}, l^{\prime}}^{b c} \rightarrow M_{k^{\prime}, l^{\prime}}^{s_{k^{\prime}}}$. Since $r f i_{k, l}\left(M_{k, l}^{s_{k-1}}\right)$ is contained in a connected subcomplex of $M_{k^{\prime}, l^{\prime}}^{s_{k^{\prime}}-1}$ generated by $N_{k, l} \leq N_{k}$ simplices, $r_{*}\left(f i_{k, l}\right)_{*}\left[M_{k, l}^{s_{k-1}}\right]=0 \in H_{k}\left(M_{k^{\prime}, l^{\prime}}^{s_{k^{\prime}-1}}\right)$ if $k^{\prime}>k$, by the choices of $s_{k}$ and $s_{k^{\prime}-1}$. If $k^{\prime}<k$, then $H_{k}\left(M_{k^{\prime}, l^{\prime}}^{s_{k^{\prime}}-1}\right)=0$ since $\operatorname{dim} M_{k^{\prime}, l^{\prime}}=k^{\prime}$. Therefore in this case we also have $r_{*}\left(f i_{k, l}\right)_{*}\left[M_{k, l}^{s_{k-1}}\right]=0 \in H_{k}\left(M_{k^{\prime}, l^{\prime}}^{s_{k^{\prime}-1}}\right)$. Since $r$ is a homotopy equivalence we conclude that if $k^{\prime} \neq k$, then $\left(f i_{k, l}\right)_{*}\left[M_{k, l}^{s_{k-1}}\right]=0 \in H_{k}\left(C_{k^{\prime}, l^{\prime}}^{b c}\right)$ and therefore $f_{*}\left(i_{k, l}\right)_{*}\left[M_{k, l}^{s_{k-1}}\right]=0 \in H_{k}(L)$. Hence the $l$-th column of $f_{*}$ is also zero. It remains to analyze the case $k^{\prime}=k$. In that case $r_{*}\left(f i_{k, l}\right)_{*}\left[M_{k, l}^{s_{k-1}}\right] \in H_{k}\left(M_{k, l^{\prime}}^{s_{k-1}}\right)$ is an integer multiple of the fundamental class $\left[M_{k, l^{\prime}}^{s_{k-1}}\right]$, and then $f_{*}\left(i_{k, l}\right)_{*}\left[M_{k, l}^{s_{k-1}}\right]=\left(i_{k, l^{\prime}}\right)_{*} r_{*}\left(f i_{k, l}\right)_{*}\left[M_{k, l}^{s_{k-1}}\right]$ is an integer multiple of $\left(i_{k, l^{\prime}}\right)_{*}\left[M_{k, l^{\prime}}^{s_{k-1}}\right]$. If $l^{\prime} \neq l$, the $l$-th column of $f_{*}$ has a zero in the $l$-th entry. The last and most important case is $l^{\prime}=l$.

If for each $k, l$ the case (1) occurs or the case $(2)$ for $\left(k^{\prime}, l^{\prime}\right) \neq(k, l)$, then the trace of the matrix of $f_{*}$ in each positive degree is zero and by the Lefschetz fixed point theorem, $f$ must fix a simplex. We can assume then that there exists one pair $k, l$ such that $(2)$ holds for $\left(k^{\prime}, l^{\prime}\right)=(k, l)$ and that $\left(f i_{k, l}\right)_{*}\left[M_{k, l}^{s_{k-1}}\right] \in H_{k}\left(C_{k, l}^{b c}\right)$ is non-trivial.

The simplicial projection $C_{k, l}^{b} \rightarrow M_{k, l}^{s_{n}}$ of the cylinder into the extreme in contact with $C_{k, l}^{c}$ extends to a simplicial retraction $p: C_{k, l}^{b c} \rightarrow C_{k, l}^{c}$. On the other hand Lemma 6 provides retractions $R_{m}: C_{*}\left(Z_{\psi_{k, l, m}}\right) \rightarrow C_{*}\left(M_{k, l}^{m}\right)$ for each $s_{k-1}<m \leq s_{n}$. Each of them extends to a retraction

$$
\widetilde{R}_{m}: C_{*}\left(\bigcup_{q=m}^{s_{n}} Z_{\psi_{k, l, q}}\right) \rightarrow C_{*}\left(\bigcup_{q=m+1}^{s_{n}} Z_{\psi_{k, l, q}}\right)
$$


When $m=s_{n}, \widetilde{R}_{m}$ is just another notation for $R_{s_{n}}$.

By Lemma 6 , the norm of the map $R_{m}$ in degree $k$ is $\left\|R_{m}\right\| \leq(k+1)$ ! for each $m$, so $\left\|\widetilde{R}_{m}\right\| \leq(k+1)$ !.

Let $c \in Z_{k}\left(M_{k, l}^{s_{k-1}}\right)$ be the fundamental cycle of $M_{k, l}^{s_{k-1}}$. Then $\|c\|$ is the number of $k$-simplices of $M_{k, l}^{s_{k-1}}$ and $p_{*}\left(f i_{k, l}\right)_{*}(c) \in Z_{k}\left(C_{k, l}^{c}\right)$ is a $k$-cycle in $C_{k, l}^{c}$. Thus

$$
\widetilde{c}=\widetilde{R}_{s_{n}} \widetilde{R}_{s_{n}-1} \ldots \widetilde{R}_{s_{k-1}+2} \widetilde{R}_{s_{k-1}+1} p_{*}\left(f i_{k, l}\right)_{*}(c) \in Z_{k}\left(M_{k, l}^{s_{n}}\right)
$$

is a cycle with norm at most $((k+1) !)^{s_{n}-s_{k-1}}\|c\|$. But this number is exactly the number of $k$-simplices in the pseudomanifold $M_{k, l}^{s_{n}}$. If $\|\widetilde{c}\|<((k+1) !)^{s_{n}-s_{k-1}}\|c\|$, the cycle $\widetilde{c}$ is carried by a proper subcomplex of $M_{k, l}^{s_{n}}$ and then it is trivial in homology. Since each $\widetilde{R}_{m}$ induces an isomorphism in homology, $f_{*}\left(i_{k, l}\right)_{*}\left[M_{k, l}^{s_{k-1}}\right]=0 \in H_{k}(L)$. This contradicts the assumption. Therefore, $\|\widetilde{c}\|=((k+1) !)^{s_{n}-s_{k-1}}\|c\|$.

Since the equality holds, we have in particular $\left\|\widetilde{R}_{s_{k-1}+1} p_{*}\left(f i_{k, l}\right)_{*}(c)\right\|=(k+1) !\|c\|$. Then for every $k$-simplex $\sigma \in M_{k, l}^{s_{k-1}}, S=p f i_{k, l}(\sigma)$ is a $k$-simplex of $C_{k, l}^{c}$ and $\left\|\widetilde{R}_{s_{k-1}+1}(S)\right\|$ $=(k+1)$ !. By Lemma 6, $S \in M_{k, l}^{s_{k-1}}$. We conclude then that $p f i_{k, l}\left(M_{k, l}^{s_{k-1}}\right) \subseteq M_{k, l}^{s_{k-1}}$ and therefore $f i_{k, l}\left(M_{k, l}^{s_{k-1}}\right) \subseteq M_{k, l}^{s_{k-1}}$. If $f i_{k, l}\left(M_{k, l}^{s_{k-1}}\right)$ is contained in a proper subcomplex of $M_{k, l}^{s_{k-1}},\left(f i_{k, l}\right)_{*}\left[M_{k, l}^{s_{k-1}}\right]=0$ and we have a contradiction. Then $\left.f\right|_{M_{k, l}^{s_{k-1}}}: M_{k, l}^{s_{k-1}} \rightarrow M_{k, l}^{s_{k-1}}$ is an automorphism and the asymmetry of $M_{k, l}^{s_{k-1}}$ gives the desired fixed simplex.

The poset of simplices of a finite simplicial complex $K$ is denoted by $\mathcal{X}(K)$. Recall that a finite poset $X$ can be regarded as a topological space with finitely many points in which open sets are those subsets $U \subseteq X$ such that any $x \in X$ which is smaller than or equal to an element of $U$ is itself in $U$. This space satisfies the $T_{0}$ separation axiom and in fact any finite $T_{0}$-space is a poset in this sense. Order preserving maps correspond to continuous maps and comparable maps are homotopic ([2]). For every finite simplicial complex $K$ there is a weak homotopy equivalence $K \rightarrow \mathcal{X}(K)$ (see [9]). The simplicial complex of chains of a poset $X$ is denoted by $\mathcal{K}(X)$. There is a weak homotopy equivalence $\mathcal{K}(X) \rightarrow X$. A simplicial map $\varphi: K \rightarrow L$ and a continuous map $f: X \rightarrow Y$ between finite $T_{0}$-spaces induce maps $\mathcal{X}(\varphi)$ and $\mathcal{K}(f)$ in the obvious way and one has the following commutative diagrams up to homotopy where the vertical maps are the weak homotopy equivalences mentioned above
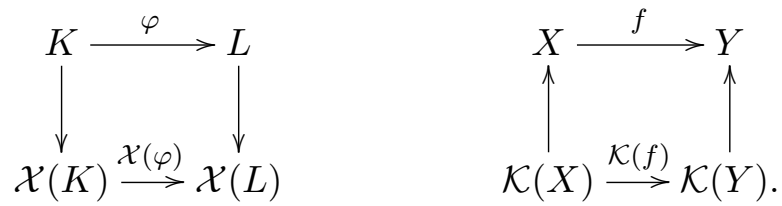

Corollary 9. Let $K$ be a simply connected compact $C W$-complex. Then there exists a topological space $X$ weak homotopy equivalent to $K$ which has the fixed point property.

Proof. By the previous theorem there is a finite simplicial complex $L$ homotopy equivalent to $K$ with the fixed simplex property. We claim that the associated finite space $\mathcal{X}(L)$ has the fixed point property. Let $f: \mathcal{X}(L) \rightarrow \mathcal{X}(L)$ be a continuous map. For every $v \in L$ choose a vertex $g(v)$ of $L$ such that $g(v) \leq f(v)$. The vertex map $g: L \rightarrow L$ is simplicial since $f$ maps a bounded set of minimal points into a bounded set. Then $g$ fixes some 
simplex $\sigma \in L$, so $\mathcal{X}(g): \mathcal{X}(L) \rightarrow \mathcal{X}(L)$ fixes $\sigma$. Since $f \geq \mathcal{X}(g), f(\sigma) \geq \sigma$ and then $f^{i}(\sigma)=f \circ f \circ \ldots \circ f(\sigma)$ is a fixed point of $f$ for $i$ large enough.

In order to extend the last corollary to non-simply connected complexes, we need to modify the construction of the space $\mathcal{X}(L)$. The idea we used in the proof of Theorem 8 fails if $H_{1}(K) \neq 0$ since no 1-dimensional pseudomanifold is asymmetric. We will adapt the proofs of Theorem 8 and Corollary 9 to the general case using the rigidity of finite spaces. Recall from $[2,3]$ that the non-Hausdorff mapping cylinder $B_{f}$ of an order preserving map $f: X \rightarrow Y$ between finite $T_{0}$-spaces is the set $X \sqcup Y$ keeping the given ordering within $X$ and $Y$ and setting $x<y$ for $x \in X$ and $y \in Y$ if $f(x) \leq y$. The cylinder $B_{f}$ deformation retracts to $Y$ so $\mathcal{K}\left(B_{f}\right)$ deformation retracts to $\mathcal{K}(Y)$ by a simplicial retraction. If $f$ is a weak homotopy equivalence, $\mathcal{K}\left(B_{f}\right)$ deformation retracts to $\mathcal{K}(X)$. If $X$ is a finite $T_{0^{-}}$ space, a point $x \in X$ such that $X_{<x}$ or $X_{>x}$ is contractible is called a weak point. In this case the inclusion $X \backslash\{x\} \hookrightarrow X$ is a weak homotopy equivalence. In other words $\mathcal{K}(X)$ deformation retracts to $\mathcal{K}(X \backslash\{x\})$ (see $[2,3]$ for more details).

Lemma 10. There exists a topological space weak homotopy equivalent to $S^{1}$ with the fixed point property.

Proof. Consider the space $\mathfrak{K}$ of 14 points in Figure 2. This space is the core of a space considered by G. Kun in [7, Remark 38] in a different context. It is constructed by gluing two non-Hausdorff mapping cylinders of 1 and 2-degree maps from an 8-point model of $S^{1}$ to a 4 -point model.

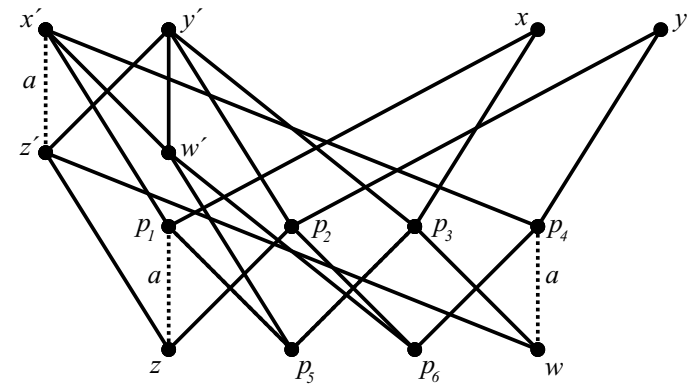

Figure 2. The space $\mathfrak{K}$, a finite model of $S^{1}$ with the fixed point property.

Since $x$ and $y$ are weak points of $\mathfrak{K}, \mathfrak{K} \backslash\{x, y\} \hookrightarrow \mathfrak{K}$ is a weak homotopy equivalence. The space $\mathfrak{K} \backslash\{x, y\}$ is a non-Hausdorff mapping cylinder and then it deformation retracts to $\left\{x^{\prime}, y^{\prime}, z^{\prime}, w^{\prime}\right\}$. Therefore $\mathfrak{K}$ is weak homotopy equivalent to $S^{1}$. We show that $\mathfrak{K}$ has the fixed point property.

We will prove the following assertion: there is, up to sign, a unique 1-cycle of norm at most 4 in the order complex $\mathcal{K}(\mathfrak{K})$ which represents the double of a generator of $H_{1}(\mathcal{K}(\mathfrak{K})) \simeq \mathbb{Z}$.

An easy way to prove this assertion is by using colorings (see [4]). The $\mathbb{Z}$-coloring of $\mathfrak{K}$ which colors the solid edges with the identity and the dotted edges with a generator $a$ of $\mathbb{Z}$ is connected and admissible, so it is the standard coloring of $\mathfrak{K}$. To each directed edge $v w$ of the complex $\mathcal{K}(\mathfrak{K})$ we assign a weight $\omega(v w)$ which is the sum of the colors of the edges in any increasing path from $v$ to $w$ if $v<w$. If $v>w, \omega(v w)=-\omega(w v)$. For example 
$\omega\left(z x^{\prime}\right)=a, \omega\left(w^{\prime} x^{\prime}\right)=0, \omega\left(p_{4} w\right)=-a$. The map $H_{1}(\mathcal{K}(\mathfrak{K})) \rightarrow \mathbb{Z}$ which maps the class of a 1-cycle $\sum v_{i} w_{i}$ to $\sum \omega\left(v_{i} w_{i}\right)$ is a well defined isomorphism (see [10, p.208] and [4]). It is now easy to check that $c=z x+x w+w y+y z$ is the unique cycle of $\mathcal{K}(\mathfrak{K})$ with norm at most 4 which corresponds to $2 a \in \mathbb{Z}$.

Alternatively, in order to prove the assertion, the reader not familiar with colorings may consider the order complex $\mathcal{K}(X)$ of the poset $X$ given by the solid edges of $\mathfrak{K}$. This complex is contractible and $\mathcal{K}(\mathfrak{K})$ is obtained from $\mathcal{K}(X)$ by adding seven 1 -simplices and six 2-simplices. Moreover, $\mathcal{K}(\mathfrak{K})$ collapses to $\mathcal{K}(X) \cup\left\{x^{\prime} z^{\prime}\right\}$ and then the homology of the 1 -cycles of $\mathcal{K}(\mathfrak{K})$ of norm 4 is easy to understand.

We use now the assertion to prove the fixed point property. Suppose $f: \mathfrak{K} \rightarrow \mathfrak{K}$ is a fixed point free map. Then $\mathcal{K}(f)$ has no fixed point and by the Lefschetz fixed point theorem $\mathcal{K}(f)_{*}: H_{1}(\mathcal{K}(\mathfrak{K})) \rightarrow H_{1}(\mathcal{K}(\mathfrak{K}))$ is the identity. Thus $\mathcal{K}(f)_{*}(c)=c$ and then $f$ maps $\{x, y, z, w\}$ into itself, so $f(x)=y, f(y)=x, f(z)=w$ and $f(w)=z$. In particular the set of points greater than $w$ and $z$ is mapped to itself, so $f\left(\left\{x^{\prime}, y^{\prime}, z^{\prime}\right\}\right) \subseteq$ $\left\{x^{\prime}, y^{\prime}, z^{\prime}\right\} \sqcup\{x\} \sqcup\{y\}$. If the connected subspace $\left\{x^{\prime}, y^{\prime}, z^{\prime}\right\}$ is mapped into the point $x$ or into $y$, then the generating cycle $z^{\prime} x^{\prime}+x^{\prime} w^{\prime}+w^{\prime} y^{\prime}+y^{\prime} z^{\prime}$ is mapped to 0 . Therefore $\left\{x^{\prime}, y^{\prime}, z^{\prime}\right\}$ is mapped into itself and then $f$ has a fixed point, a contradiction.

Proof of Theorem 1. We may suppose that $K$ is a finite simplicial complex. We begin with the construction of $L$ performed in the proof of Theorem 8, except that this time we consider also integer homology classes $\alpha_{1,1}, \alpha_{1,2}, \ldots, \alpha_{1, d_{1}}$ which are a basis for $H_{1}(K ; \mathbb{Q})$ and 1-pseudomanifolds $M_{1, l}$ along with simplicial maps $\varphi_{1, l}: M_{1, l} \rightarrow K$ which map [ $\left.M_{1, l}\right]$ to $\alpha_{1, l}$. The pseudomanifolds $M_{k, l}$ will be assumed to be asymmetric for $k \geq 2$, but of course this is not possible for $k=1$.

The numbers $s_{1}, N_{k, l}, N_{k}, s_{k}$ for $k \geq 2$ are defined as before. Let $s_{0}=0, N_{1}=1$ and $N=\max _{1 \leq k \leq n} N_{k}$. The cylinders $C_{k, l}$ are built just as before, except that $C_{k, l}^{b}$ will be constructed by gluing not $N$ cylinders but $(n+1) ! N$ cylinders of the identity. Also, we include now the $C_{1, l}$ 's. The complex $L$ is the union of all these cylinders and it is homotopy equivalent to $K$. The unique difference was the incorporation of the 1dimensional manifolds with their cylinders and that we increased the length of the cylinders $C_{k, l}^{b}$.

The space $X$ will contain $\mathcal{X}(L)$ as a subspace. For each $1 \leq l \leq d_{1}$ consider a weak homotopy equivalence $\mathcal{X}\left(M_{1, l}\right) \rightarrow\left\{x^{\prime}, y^{\prime}, z^{\prime}, w^{\prime}\right\}$ where the codomain is the subspace of $\mathfrak{K}$ defined in Lemma 10. Since $\left\{x^{\prime}, y^{\prime}, z^{\prime}, w^{\prime}\right\} \hookrightarrow \mathfrak{K}$ is a weak equivalence, the composition $h_{l}: \mathcal{X}\left(M_{l, 1}\right) \rightarrow \mathfrak{K}$ is a weak equivalence. We take a different copy of $\mathfrak{K}$ for each $1 \leq l \leq d_{1}$, so the non-Hausdorff mapping cylinders $B_{h_{l}}$ are disjoint. Let $X=\mathcal{X}(L) \cup \bigcup_{l=1}^{d_{1}} B_{h_{l}}$. Since $h_{l}$ is a weak equivalence, $\mathcal{K}\left(B_{h_{l}}\right)$ deformation retracts to $\mathcal{K}\left(\mathcal{X}\left(M_{1, l}\right)\right)=M_{1, l}^{\prime}$ and then $\mathcal{K}(X)$ deformation retracts to $L^{\prime}$. Therefore $X$ is weak homotopy equivalent to $K$. We prove that $X$ has the fixed point property.

Let $f: X \rightarrow X$ be a continuous map. Then $\mathcal{K}(f): \mathcal{K}(X) \rightarrow \mathcal{K}(X)$ is a simplicial map. If $\mathcal{K}(f)$ fixes a simplex, then $f$ fixes a chain, so $f$ fixes all the elements of the chain. For each $k \geq 1$ we consider the basis $\mathcal{B}_{k}=\left\{\left(i_{k, l}^{\prime}\right)_{*}\left(\left[M_{k, l}^{s_{k-1}+1}\right]\right) \otimes 1_{\mathbb{Q}}\right\}_{l}$ of $H_{k}(\mathcal{K}(X) ; \mathbb{Q})$. Let $k \geq 2$ and $1 \leq l \leq d_{k}$. Then $\mathcal{K}(f)\left(M_{k, l}^{s_{k-1}+1}\right)$ lies in a complex generated by $(k+1) !\left\|\left[M_{k, l}^{s_{k-1}}\right]\right\| \leq$ $(k+1) ! N_{k} \leq(n+1) ! N$ simplices. We consider now three cases in order to take into account the 1-dimensional part: 
(1) $\mathcal{K}(f)\left(M_{k, l}^{s_{k-1}+1}\right) \subseteq\left(C^{a b}\right)^{\prime}$ or

(2) $\mathcal{K}(f)\left(M_{k, l}^{s_{k-1}+1}\right) \subseteq\left(C_{k^{\prime}, l^{\prime}}^{b c}\right)^{\prime}$ for some $k^{\prime} \geq 2$ and some $l^{\prime}$ or

(3) $\mathcal{K}(f)\left(M_{k, l}^{s_{k-1}+1}\right) \subseteq \mathcal{K}\left(\mathcal{X}\left(C_{1, l^{\prime}}^{b c}\right) \cup B_{h_{l^{\prime}}}\right)$ for some $l^{\prime}$.

In the third case, since $\mathcal{K}\left(\mathcal{X}\left(C_{1, l^{\prime}}^{b c}\right) \cup B_{h_{l^{\prime}}}\right)$ deformation retracts to $\left(C_{1, l^{\prime}}^{b c}\right)^{\prime}$ which in turn deformation retracts into the 1 -dimensional complex $M_{1, l^{\prime}}^{\prime}$, then $\mathcal{K}(f)_{*}\left(i_{k, l}^{\prime}\right)_{*}\left[M_{k, l}^{s_{k-1}+1}\right]=$ $0 \in H_{k}(\mathcal{K}(X))$.

If (1) holds, $f\left(\mathcal{X}\left(M_{k, l}^{s_{k-1}}\right)\right) \subseteq \mathcal{X}\left(C^{a b}\right)$. As in the proof of Corollary $9, f: \mathcal{X}\left(M_{k, l}^{s_{k-1}}\right) \rightarrow$ $\mathcal{X}\left(C^{a b}\right)$ induces a simplicial map $g: M_{k, l}^{s_{k-1}} \rightarrow C^{a b}$ which is zero in $H_{k}$ by the proof of Theorem 8. Then $\mathcal{X}(g): \mathcal{X}\left(M_{k, l}^{s_{k-1}}\right) \rightarrow \mathcal{X}\left(C^{a b}\right)$ is zero in $k$-homology and the same is true for $f: \mathcal{X}\left(M_{k, l}^{s_{k-1}}\right) \rightarrow \mathcal{X}\left(C^{a b}\right)$ since this restriction of $f$ is homotopic to $\mathcal{X}(g)$. Then $\mathcal{K}(f): M_{k, l}^{s_{k-1}+1} \rightarrow\left(C^{a b}\right)^{\prime}$ induces the trivial map so $\mathcal{K}(f)_{*}\left(i_{k, l}^{\prime}\right)_{*}\left[M_{k, l}^{s_{k-1}+1}\right]=0$. This idea works also to adapt the proof of Theorem 8 to the cases (2) for $k^{\prime}<k$, (2) for $k^{\prime}>k,(2)$ for $k^{\prime}=k$ and $l^{\prime} \neq l$. Also, if we are in the case (2) with $k^{\prime}=k, l^{\prime}=l$ then $f: \mathcal{X}\left(M_{k, l}^{s_{k-1}}\right) \rightarrow \mathcal{X}\left(C_{k, l}^{b c}\right)$ induces $g: M_{k, l}^{s_{k-1}} \rightarrow C_{k, l}^{b c}$. If $\mathcal{K}(f)_{*}\left(i_{k, l}^{\prime}\right)_{*}\left[M_{k, l}^{s_{k-1}+1}\right] \neq 0$, $g_{*}\left[M_{k, l}^{s_{k-1}}\right] \neq 0$ and then by the proof of Theorem $8 g$ fixes a simplex, so $\mathcal{X}(g)$ fixes a point $\sigma \in \mathcal{X}\left(M_{k, l}^{s_{k-1}}\right)$. Then $f(\sigma) \geq \mathcal{X}(g)(\sigma)=\sigma$ and $f: X \rightarrow X$ has a fixed point.

We can then assume that the trace of $\mathcal{K}(f)_{*}: H_{k}(\mathcal{K}(X) ; \mathbb{Q}) \rightarrow H_{k}(\mathcal{K}(X) ; \mathbb{Q})$ is zero for each $k \geq 2$.

Now we study the 1-dimensional component. Let $1 \leq l \leq d_{1}$. Then $\{x, y, z, w\} \subseteq$ $B_{h_{l}}$ and if $j_{l}:\{x, y, z, w\} \hookrightarrow X$ denotes the inclusion which factors through $B_{h_{l}}$ and $\beta_{l}=[\mathcal{K}(\{x, y, z, w\})]$ denotes the fundamental class of $\mathcal{K}(\{x, y, z, w\})$, then $\mathcal{K}\left(j_{l}\right)_{*}\left(\beta_{l}\right)=$ $2\left(i_{1, l}^{\prime}\right)_{*}\left[M_{1, l}^{\prime}\right] \in H_{1}(\mathcal{K}(X))$. Thus $\mathcal{K}\left(j_{l}\right)_{*}\left(\beta_{l}\right) \otimes 1_{\mathbb{Q}}$ is twice an element of $\mathcal{B}_{1}$, the chosen basis for $H_{1}(\mathcal{K}(X) ; \mathbb{Q})$.

Since $(n+1) ! N \geq 1$, for each $1 \leq l \leq d_{1}$ the $l$-th copy of $\mathcal{K}(\{x, y, z, w\})$ is mapped by $\mathcal{K}(f)$ into $L^{\prime}$ or into $\mathcal{K}\left(\mathcal{X}\left(C_{1, l^{\prime}}^{b c}\right) \cup B_{h_{l^{\prime}}}\right)$ for some $l^{\prime}$.

If $\mathcal{K}(f)(\mathcal{K}(\{x, y, z, w\})) \subseteq L^{\prime}$, then it is contained in a contractible subcomplex since any closed edge-path of four edges in a barycentric subdivision is contained in the star of a vertex, and then $\mathcal{K}(f)_{*} \mathcal{K}\left(j_{l}\right)_{*}\left(\beta_{l}\right)=0 \in H_{1}(\mathcal{K}(X))$. If $\mathcal{K}(f)(\mathcal{K}(\{x, y, z, w\}))$ is contained in $\mathcal{K}\left(\mathcal{X}\left(C_{1, l^{\prime}}^{b c}\right) \cup B_{h_{l^{\prime}}}\right)$ for some $l^{\prime} \neq l$, then $\mathcal{K}\left(f j_{l}\right): \mathcal{K}(\{x, y, z, w\}) \rightarrow \mathcal{K}\left(\mathcal{X}\left(C_{1, l^{\prime}}^{b c}\right) \cup B_{h_{l^{\prime}}}\right)$. The codomain of this map deformation retracts to $M_{1, l^{\prime}}^{\prime}$ by a retraction $r$ (which is not simplicial). Then $i_{1, l^{\prime}}^{\prime} r \mathcal{K}\left(f j_{l}\right): \mathcal{K}(\{x, y, z, w\}) \rightarrow \mathcal{K}(X)$ is homotopic to $\mathcal{K}(f) \mathcal{K}\left(j_{l}\right)$ and therefore $\mathcal{K}(f)_{*} \mathcal{K}\left(j_{l}\right)_{*}\left(\beta_{l}\right)$ is an integer multiple of $\left(i_{1, l^{\prime}}^{\prime}\right)_{*}\left[M_{1, l^{\prime}}^{\prime}\right]$. In any of the cases considered so far, the matrix of $\mathcal{K}(f)_{*}$ in the basis $\mathcal{B}_{1}$ has a zero in the entry $(l, l)$. Suppose then that $\mathcal{K}(f)(\mathcal{K}\{x, y, z, w\})$ is contained in $\mathcal{K}\left(\mathcal{X}\left(C_{1, l}^{b c}\right) \cup B_{h_{l}}\right)$. Since $C_{1, l}^{b c}$ deformation retracts to $M_{1, l}$ by a simplicial retraction, $\mathcal{K}\left(\mathcal{X}\left(C_{1, l}^{b c}\right) \cup B_{h_{l}}\right)$ deformation retracts to $\mathcal{K}\left(B_{h_{l}}\right)$ by a simplicial retraction. Moreover, $\mathcal{K}\left(B_{h_{l}}\right)$ deformation retracts to $\mathcal{K}(\mathfrak{K})$ by a simplicial retraction which maps $M_{1, l}^{\prime}$ into $\mathcal{K}\left(\left\{x^{\prime}, y^{\prime}, z^{\prime}, w^{\prime}\right\}\right)$. Then $\mathcal{K}\left(\mathcal{X}\left(C_{1, l}^{b c}\right) \cup B_{h_{l}}\right)$ deformation retracts to $\mathcal{K}(\mathfrak{K})$ by a simplicial retraction $R: \mathcal{K}\left(\mathcal{X}\left(C_{1, l}^{b c}\right) \cup B_{h_{l}}\right) \rightarrow \mathcal{K}(\mathfrak{K})$ which maps $\left(C_{1, l}^{b c}\right)^{\prime}$ into $\mathcal{K}\left(\left\{x^{\prime}, y^{\prime}, z^{\prime}, w^{\prime}\right\}\right)$. Thus, $\mathcal{K}(f)_{*} \mathcal{K}\left(j_{l}\right)_{*}\left(\beta_{l}\right)=i_{*} R_{*} \mathcal{K}\left(f j_{l}\right)_{*}\left(\beta_{l}\right)$ where $i: \mathcal{K}(\mathfrak{K}) \rightarrow \mathcal{K}(X)$ denotes the inclusion. Then the homology class $\mathcal{K}\left(j_{l}\right)_{*}\left(\beta_{l}\right)=2\left(i_{1, l}^{\prime}\right)_{*}\left[M_{1, l}^{\prime}\right]$ is mapped by $\mathcal{K}(f)$ to an integer multiple of the generator $\left(i_{1, l}^{\prime}\right)_{*}\left[M_{1, l}^{\prime}\right]$ of the image of $i_{*}$. Therefore, $\mathcal{K}\left(j_{l}\right)_{*}\left(\beta_{l}\right)$ is mapped to an integer multiple of itself. Since $f$ is a self map of a finite set, the powers 
of $f$ induce only finite morphisms in homology, so $\mathcal{K}\left(j_{l}\right)_{*}\left(\beta_{l}\right)$ is mapped to $0, \mathcal{K}\left(j_{l}\right)_{*}\left(\beta_{l}\right)$ or $-\mathcal{K}\left(j_{l}\right)_{*}\left(\beta_{l}\right)$. By the Lefschetz fixed point theorem we can assume that for some $1 \leq l \leq d_{1}$, $\mathcal{K}\left(j_{l}\right)_{*}\left(\beta_{l}\right)$ is mapped to itself. Then $R_{*} \mathcal{K}\left(f j_{l}\right)_{*}(z x+x w+w y+y z) \in Z_{1}(\mathcal{K}(\mathfrak{K}))$ is a 1 -cycle of norm at most 4 which represents the double of the generator of $H_{1}(\mathcal{K}(\mathfrak{K}))$. By the assertion in Lemma 10 there is a unique cycle satisfying these conditions, which is $z x+x w+w y+y z$. Therefore $R \mathcal{K}\left(f j_{l}\right)$ maps $\{x, y, z, w\}$ into itself and then $f(\{x, y, z, w\})=\{x, y, z, w\}$. Thus, the set of points smaller than one of those four points, $\left\{x, y, z, w, p_{1}, p_{2}, \ldots, p_{6}\right\}$, is mapped also to itself and then $f$ maps $\mathfrak{K}$ into $\mathfrak{K}$. By Lemma $10, f$ has a fixed point.

\section{REFERENCES}

[1] K. Baclawski, A. Björner. Fixed points in partially ordered sets. Adv. Math. 31(1979), 263-287.

[2] J.A. Barmak. Algebraic topology of finite topological spaces and applications. Lecture Notes in Mathematics 2032, Springer, Heidelberg, 2011. xviii+170 pp.

[3] J.A. Barmak, E.G. Minian. Simple homotopy types and finite spaces. Adv. Math. 218(2008), Issue 1, 87-104.

[4] J.A. Barmak, E.G. Minian. G-colorings of posets, coverings and presentations of the fundamental group. arXiv: 1212.6442

[5] A. Hatcher. Algebraic Topology. Cambridge University Press, Cambridge, 2002. xii+544 pp.

[6] B. Jiang. On the least number of fixed points. Amer. J. Math. 102(1980), 749-763.

[7] G. Kun. On the fundamental group of posets. Master Thesis, Eötvös Loránd University (2003).

[8] W. Lopez. An example in the fixed point theory of polyhedra. Bull. Amer. Math. Soc. 73(1967), 922-924.

[9] M.C. McCord. Singular homology groups and homotopy groups of finite topological spaces. Duke Math. J. 33(1966) 465-474.

[10] E. Spanier. Algebraic Topology. McGraw-Hill Book Co., New York-Toronto, Ont.-London 1966 xiv+528 pp.

[11] R. Waggoner. A fixed point theorem for $(n-2)$-connected n-polyhedra. Proc. Amer. Math. Soc. 33(1972), 143-145.

Departamento de Matematica, FCEyn-Universidad de Buenos Aires, Buenos Aires, ArGENTINA

E-mail address: jbarmak@dm.uba.ar 